\title{
BILATERAL INTRA-RETINAL MACULAR HAEMORRHAGE IN A YOUNG FEMALE, A RARE PRESENTATION: A CASE REPORT
}

\author{
Inderjit Kaur ${ }^{1}$, Prempal Kaur², Mona Tuli³, Shaurya Sharma ${ }^{4}$
}

\section{HOW TO CITE THIS ARTICLE:}

Inderjit Kaur, Prempal Kaur, Mona Tuli, Shaurya Sharma. "Bilateral Intra-Retinal Macular Hemorrhage in a Young Female, a Rare Presentation: A Case Report". Journal of Evolution of Medical and Dental Sciences 2014; Vol. 3, Issue 05, February 03; Page: 1276-1283, DOI: 10.14260/jemds/2014/1980

ABSTRACT: AIM: To report a case of bilateral intra-retinal macular hemorrhage in a young female. CASE REPORT: A 25 year old healthy female, with no history of prior ocular complaints presented to the Regional Institute of Ophthalmology Medical College Amritsar with the complaint of sudden bilateral painless blurring of vision since 24 hours. On examination, visual acuity was 6/36 partial in OD and 6/60 partial in OS. Near vision was 6/12 partial OU. Fundoscopy disclosed bilateral maculopathic hemorrhage confirmed with OCT. Investigations for the common causes such as drug induced anemia, blood dyscrasias, diabetes, hypertension, vasculitides and valsalva retinopathy were carried out. A platelet count of 1,00,000 was the only significant finding. All other results were normal. On the basis of clinical suspicion and low platelet count, Dengue Serology was advised which was positive. Ruling out other causes, a diagnosis of Dengue Maculopathy causing bilateral intraretinal hemorrhage was formed. Patient was kept under observation, and showed drastic improvement. CONCLUSIONS: In literature, bilateral intra-retinal macular hemorrhage alone is a rare finding. Apart from the more common causes, dengue fever should be kept in mind. Dengue associated maculopathy most commonly presents as blurring of vision with macular hemorrhage or macular edema. Hence in endemic areas, a high index of suspicion and thorough ocular examination is mandatory in dengue suspects.

KEYWORDS: Bilateral, intra-retinal, macular haemorrhage, dengue fever.

INTRODUCTION: Bilateral intra-retinal macular hemorrhage alone is a rare ocular finding. Cases reported with this presentation alone are few in literature. The probable differentials of such a presentation can be drug-induced anemia, trauma, welding arc exposure, post corneal or refractive surgery e.g. LASIK, idiopathic recurrent phlebitis, blood dyscrasias including auto immune hemolytic anemia, thrombocytopenia, vasculitides, hypertension, Diabetes Mellitus, dengue fever and valsalva retinopathy. Definitive diagnosis can be made after a comprehensive history, thorough systemic evaluation and complete ophthalmological examination along with investigations such as fundus photography, visual fields, amsler grid test, FFA, ICG and OCT.

CASE REPORT: A 25 year old female from a village in Amritsar district, housewife and a mother of two, with no history of any prior ocular complaints presented at the Regional Institute of Ophthalmology, Amritsar OPD with the complaint of sudden painless bilateral blurring of vision. There was no history of fever, diarrhoea, vomiting, any head or ocular trauma. There was no history of any systemic affliction like diabetes, hypertension, any vasculitides, blood dyscrasias, blood transfusions, any ocular surgical intervention or drug intake.

History of present illness: Patient complained of blurring of vision in both eyes since 24 hours before which patient was apparently asymptomatic.

On general examination patient was calm, cooperative and conscious. 


\section{CASE REPORT}

Vitals: Blood Pressure: 120/74 mm of Hg, Pulse: 70/minute, Afebrile.

- VA: 6/36 partial OD and 6/60 partial OS

- No improvement with pin hole

- Near Vision: N12 partial OU

- Pupillary responses and intraocular pressure were normal

- Anterior segment WNL

- Fundus examination showed the following picture:

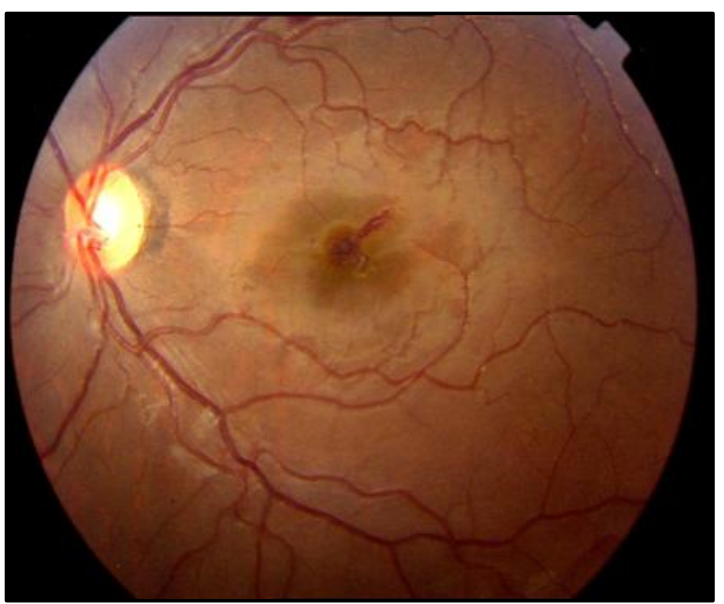

FUNDUS PICTURE OD: MACULAR HAEMORRHAGE

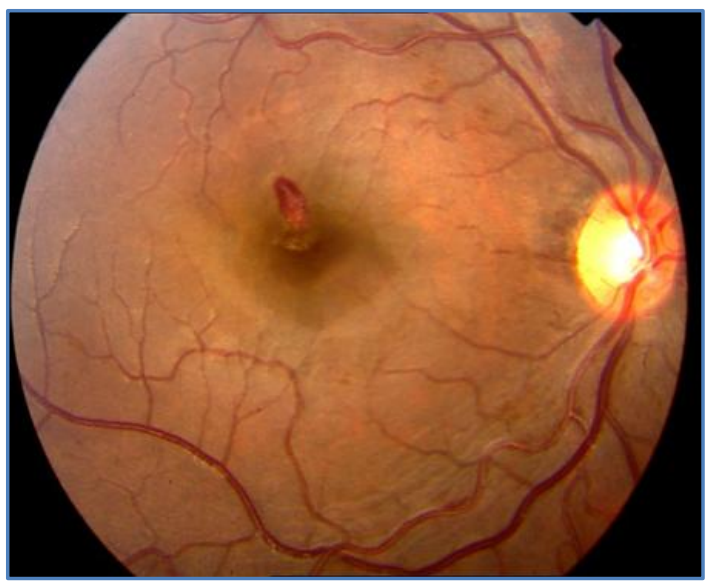

FUNDUS PICTURE OS: MACULAR HAEMORRHAGE 


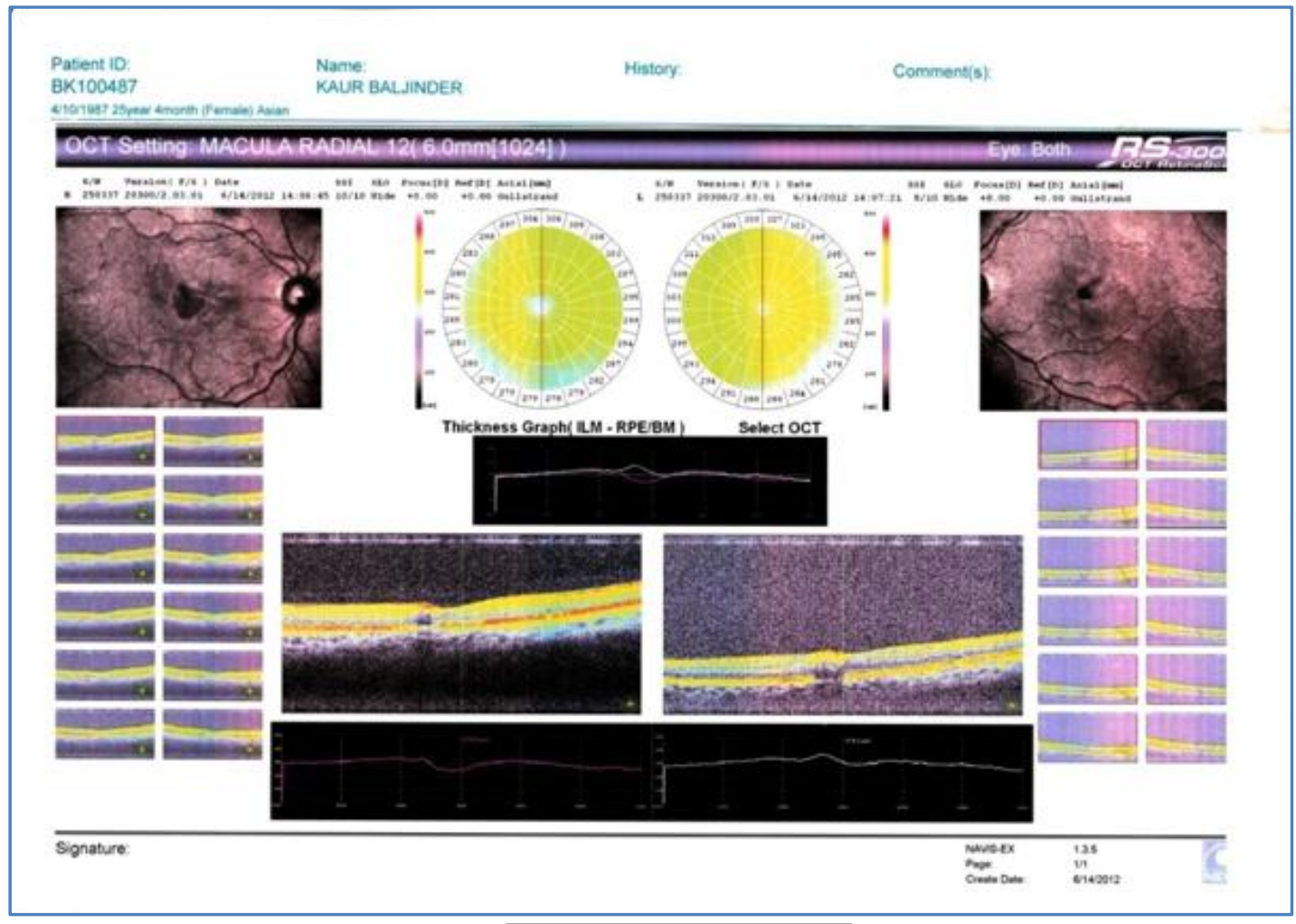

OCT OF PATIENT
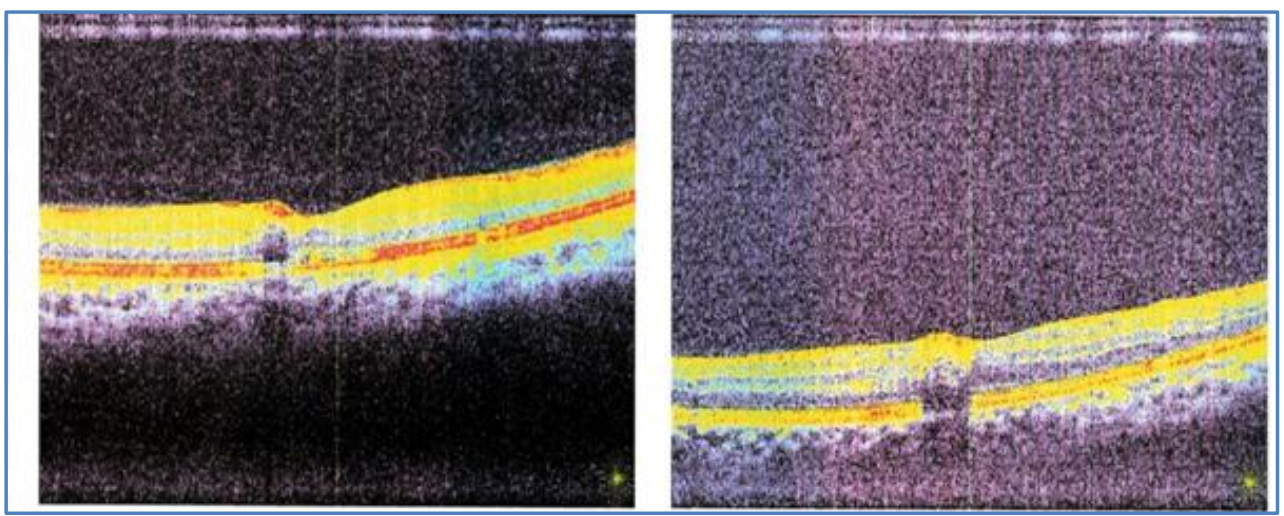

OCT ODOCT OU

OCT OU showed intraretinal macular hemorrhages.

Patient was advised to instill NSAID eye drops and prescribed oral antioxidants till other reports were awaited. Systemic investigations were within normal limits except for a low platelet count $\left(1,00,000 / \mathrm{mm}^{3}\right)$. On the basis of clinical suspicion and a low seemingly low platelet count, we advised the patient dengue serology- which came out positive- which came out POSITIVE.

Patient was treated and managed for dengue in Guru Nanak Dev Hospital and was followed up in the Ophthalmology Out-patient department. 


\section{CASE REPORT}

Conservative treatment was continued for her ophthalmic complaints and follow up included repeat OCT and HVF.

Patient's visual acuity improved to $6 / 12$ partial OU on follow up after two months, OCT also showed improvement and resolution of the hemorrhage.

\section{TWO MONTHS POST EPISODE}

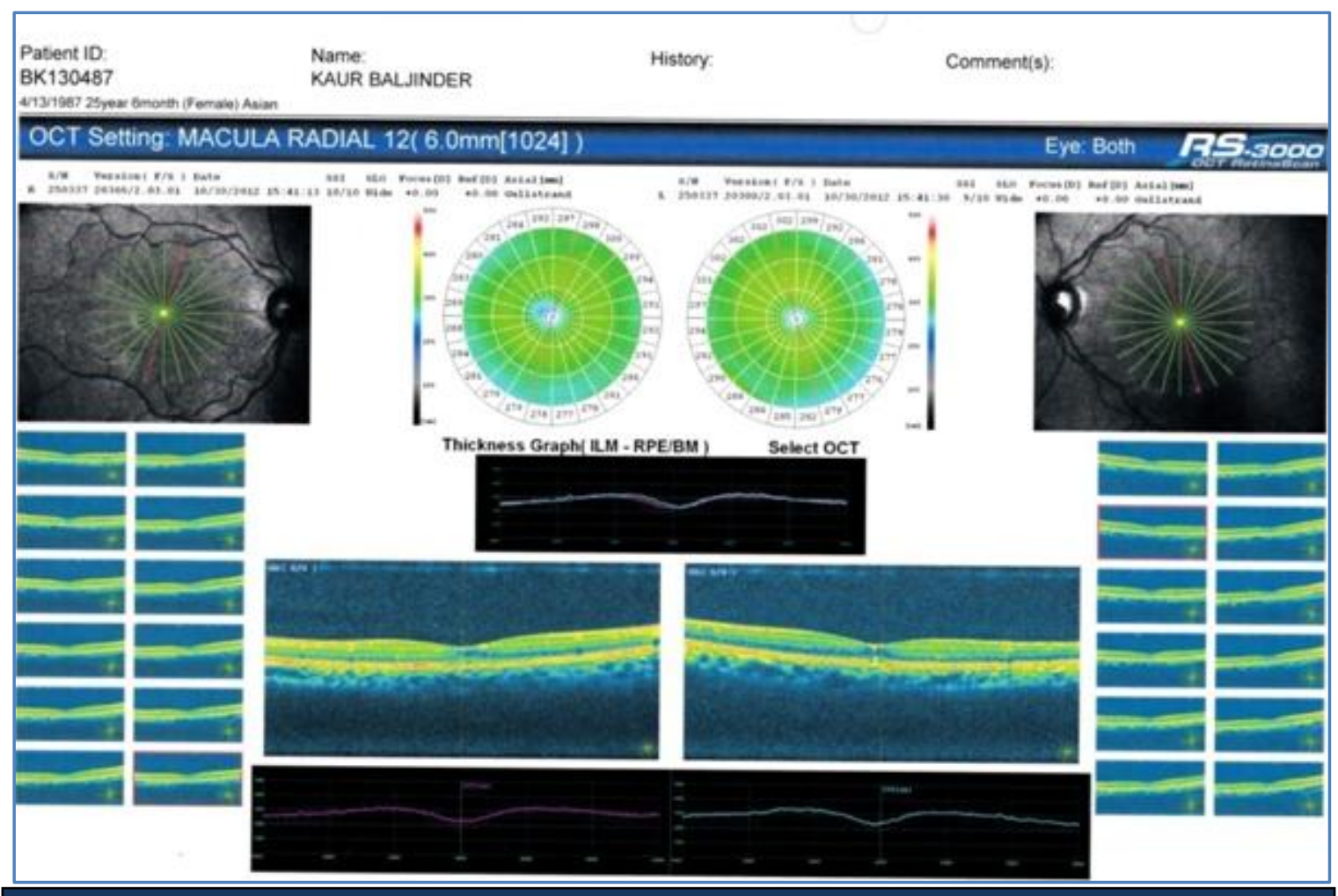

IMPROVEMENT SEEN ON OCT POST TWO MONTHS OF CONSERVATIVE TREATMENT 
CASE REPORT

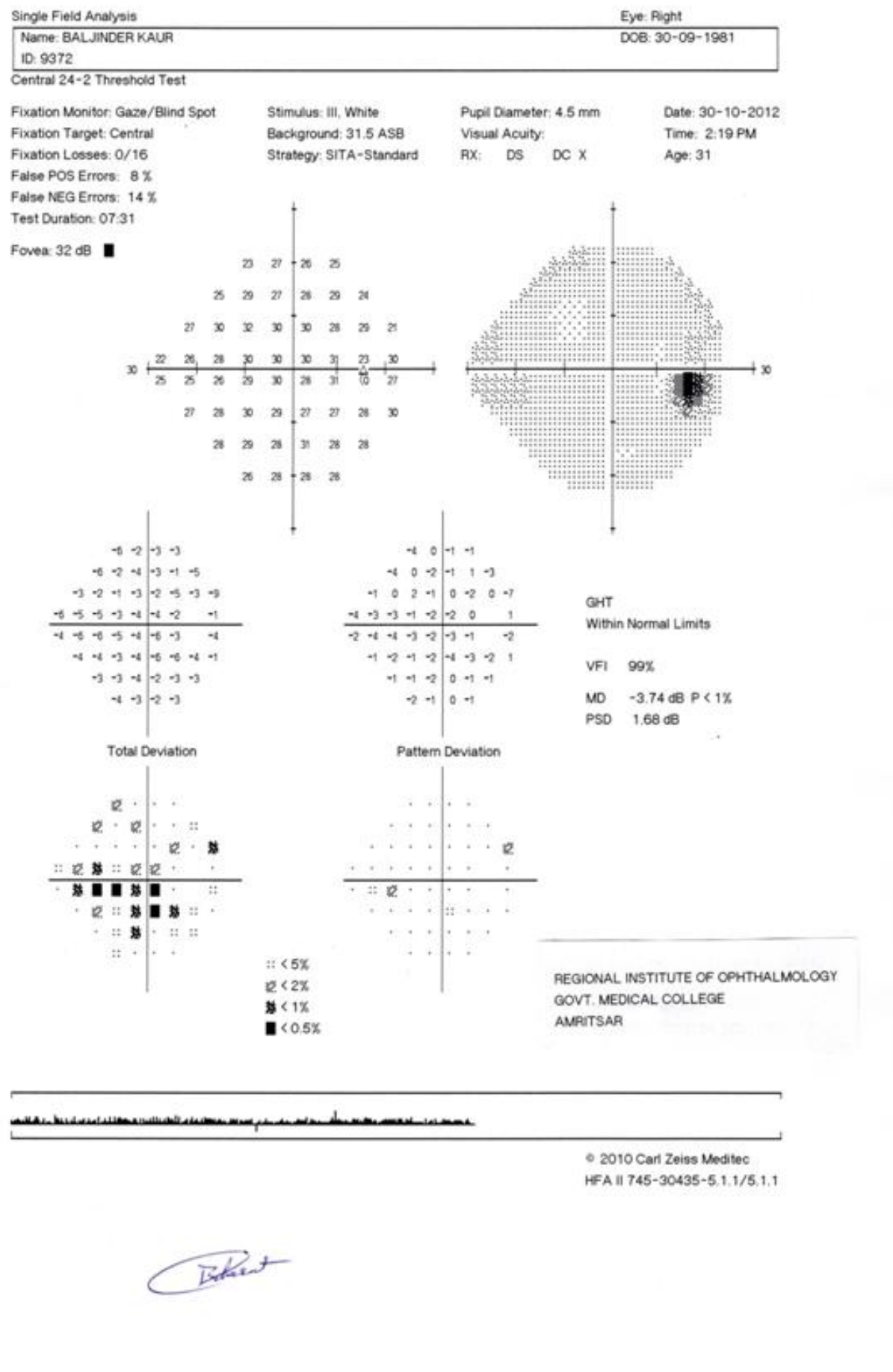

OD HVF 24-2 shows resolution two months post-episode 


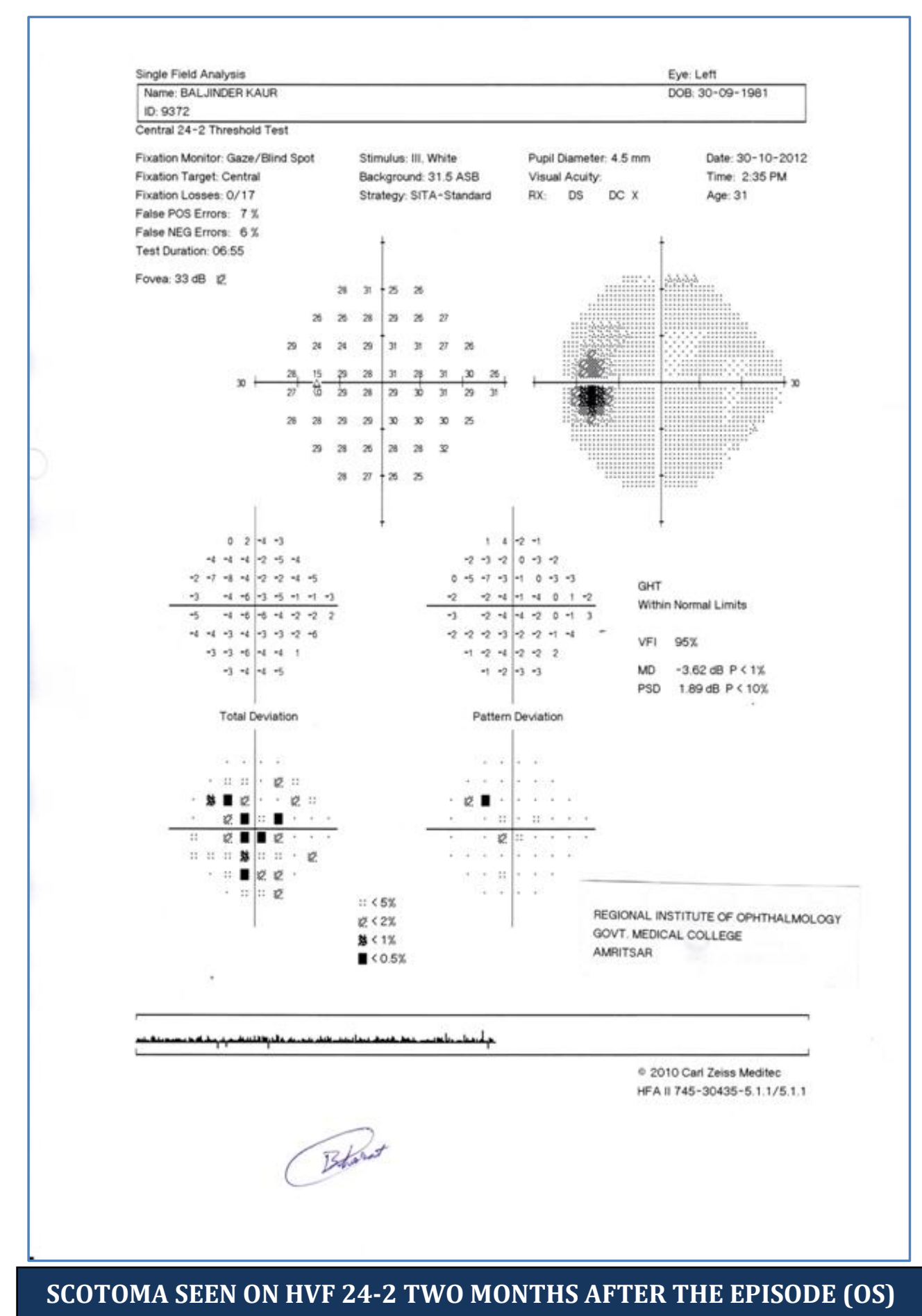

DISCUSSION: Dengue fever, the most common mosquito borne viral disease in humans is a multisystemic disease with known ocular complications. ${ }^{1}$ The typical patient is a young immunocompetent individual with a history of travel to, or residence in an endemic area, who presents with visual blurring or paracentral scotoma on days 5 to 7 after onset of fever coinciding with the nadir of thrombocytopenia. ${ }^{2}$ Dengue maculopathy which could be caused by vascular alterations and/or aberrant immune response after infection may result in temporary or permanent visual losses. 
Dengue fever can lead to visual impairment in the form of blurring of vision (most common), central scotoma, micropsia/metamorphosia, floaters, visual field defect, near vision disturbance, impairment of color vision, ocular pain and redness visual field defect, detectable by ophthalmological exams such as angiography, retinography and OCT imaging, as well as retinal and cortical electrophysiology. The Snellen Visual Acuity varies from 6/6 to counting fingers only (median 6/12) and is maculopathy dependent. A significant improvement in mean BCVA from baseline is noted between weeks 2 and 4 and may take upto three months. Although visual acuity continues to improve thereafter, the difference between subsequent follow-up intervals is less significant. ${ }^{3}$

Ocular complications involving the anterior segment of the eye include subconjunctival hemorrhage, anterior uveitis and dengue-related shallow anterior chamber. Ocular complications of the posterior segment of the eye are most commonly found on the macular region. Dengue maculopathy has myriad manifestations: inflammatory and occlusive or both. Macular hemorrhage and edema make up the majority of the findings. Ocular symptoms manifest at or close to the trough of the serum platelets and leucocytes level as well as 5 to 7 days from the onset of dengue fever. This parallels the proposed immune-mediated inflammatory mechanism of dengue maculopathy. Another mechanism proposed is the thrombocytopenic state resulting in bleeding tendencies and retinal haemorrhages. ${ }^{4}$ Teoh et al observed that the onset of manifestations coinciding with the start of thrombocytopenia recovery correlates with increased immunological response, hence supporting the immune medicated mechanism of pathogenesis. ${ }^{5}$ Hemorrhages associated with dengue-related maculopathy are mostly intraretinal and can take the form of dot, blot, or flame-shaped hemorrhages.

Ophthalmic investigations are performed mostly for posterior segment pathology and include visual acuity, slit-lamp examination, dilated fundus examination, visual fields, Amsler Grid test, FFA, ICG and OCT.2 Prognosis is generally good as the disease is often self-limiting, resolving spontaneously even without treatment. Patients may experience mild relative central scotoma that may persist for months. ${ }^{1}$ The use of steroids in treating this inflammatory eye condition is controversial. In conclusion, a high index of suspicion is necessary in cases reported in endemic areas and in people with a recent history of travel to the same in order to diagnose Dengue as a cause of ophthalmic manifestations.

\section{REFERENCES:}

1. Bascal KE, Chee SP, Cheng CL, Flores JV. Dengue-associated maculopathy. Arch Ophthalmol. 2007; 125:501-510.

2. Chlebicki MP, Ang B, Barkham T, Laude A. Retinal hemorrhages in four patients with dengue fever. Emerg Infect Dis. 2005, May; 11(5):770-2.

3. Teoh SCB, Chan DPL, Nah GKM, Rajagopalan R, Laude A, Ang B et al. The Eye Institute Denguerelated Ophthalmic Complication Workgroup. A re-look at ocular complications in Dengue fever and Dengue haemorrhagic fever. Dengue Bulletin 2006, (30):184-90. Available at < http://209.61.208.233/LinkFiles/Dengue_Bulletins_c23.pdf>

4. Young SM, Santiago P, Chee CKL. A Patient Presenting with Bilateral Central Scotomas after Dengue Fever. World J Retina Vitreous 2012; 2(1):18-21.

5. Lim WK, Mathur R, Koh A, Yeoh R, Chee SP. Ocular manifestations of dengue fever. Ophthalmology 2004; 111:2057-64. 


\section{CASE REPORT}

\section{AUTHORS:}

1. Inderjit Kaur

2. Prempal Kaur

3. Mona Tuli

4. Shaurya Sharma

\section{PARTICULARS OF CONTRIBUTORS:}

1. Associate Professor, Department of Ophthalmology, Regional Institute of Ophthalmology, GMC, Amritsar, Punjab.

2. Associate Professor, Department of Ophthalmology, Regional Institute of Ophthalmology, GMC, Amritsar, Punjab.

3. Senior Resident, Department of Ophthalmology, Regional Institute of Ophthalmology, GMC, Amritsar, Punjab.
4. Junior Resident, Department of Ophthalmology, Regional Institute of Ophthalmology, GMC, Amritsar, Punjab.

\section{NAME ADDRESS EMAIL ID OF THE CORRESPONDING AUTHOR:}

Dr.Inderjit Kaur, 20, Doctor's Avenue, Majitha Road, Amritsar - 143001, Punjab.

E-mail: renudoc@yahoo.com

Date of Submission: 30/12/2013.

Date of Peer Review: 01/01/2014.

Date of Acceptance: 16/01/2014.

Date of Publishing: 30/01/2014. 